\title{
Algebraic models of the Euclidean plane
}

\author{
Jérémy Blanc and Adrien Dubouloz
}

\begin{abstract}
We introduce a new invariant, the real (logarithmic)-Kodaira dimension, that allows to distinguish smooth real algebraic surfaces up to birational diffeomorphism. As an application, we construct infinite families of smooth rational real algebraic surfaces with trivial homology groups, whose real loci are diffeomorphic to $\mathbb{R}^{2}$, but which are pairwise not birationally diffeomorphic. There are thus infinitely many non-trivial models of the euclidean plane, contrary to the compact case.
\end{abstract}

Keywords. Real algebraic model; affine surface; rational fibration; birational diffeomorphism; affine complexification

2010 Mathematics Subject Classification. 14R05; 14R25; 14E05; 14P25; 14J26

\section{[Français]}

Titre. Modèles algébriques du plan euclidien

Résumé. Nous introduisons un nouvel invariant, la dimension de Kodaira (logarithmique) réelle, qui permet de distinguer les surfaces algébriques réelles lisses à difféomorphismes birationnels près. En guise d'application, nous construisons des familles infinies de surfaces algébriques réelles rationnelles lisses ayant des groupes d'homologie triviaux, dont les lieux réels sont difféomorphes à $\mathbb{R}^{2}$ mais qui sont deux à deux non birationnellement difféomorphes. Contrairement au cas compact, il y a donc une infinité de modèles non triviaux du plan euclidien.

Received by the Editors on May 16, 2018, and in final form on October 10, 2018. Accepted on November 13, 2018.

Jérémy Blanc

Departement Mathematik und Informatik, Unversität Basel, Spiegelgasse 1, CH-4051 Basel, Switzerland

e-mail: jeremy.blanc@unibas.ch

Adrien Dubouloz

IMB UMR5584, CNRS, Univ. Bourgogne Franche-Comté, F-21000 Dijon, France

e-mail: adrien.dubouloz@u-bourgogne.fr

The first author acknowledges support by the Swiss National Science Foundation Grant "Birational Geometry" PP00P2_153026. 


\section{Contents}

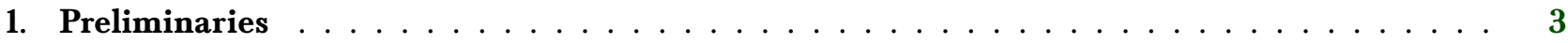

2. The real Kodaira dimension of open real surfaces $\ldots \ldots \ldots \ldots$

3. Families of algebraic models of Kodaira dimension $1 \ldots \ldots \ldots$

4. Families of algebraic models of Kodaira dimensions $2 \ldots \ldots \ldots \ldots \ldots$

\section{Introduction}

A real quasi-projective algebraic variety $X$ can be viewed as a complex quasi-projective algebraic variety endowed with an anti-regular involution, or equivalently as a locally closed subscheme of $\mathbb{P}_{\mathbb{C}}^{n}$ which is defined over $\mathbb{R}$. We can then speak about the set $X(\mathbb{R})$ of real points of $X$ (real locus). If $X$ is smooth, this set is naturally endowed with the structure of differential real manifold, and $X$ is said to be an algebraic model of this differential manifold. Two models $X_{1}$ and $X_{2}$ of the same manifold are said to be equivalent if there exists a diffeomorphism $X_{1}(\mathbb{R}) \rightarrow X_{2}(\mathbb{R})$ which comes from a birational map $\varphi: X_{1} \rightarrow X_{2}$, such that $\varphi$ and $\varphi^{-1}$ are defined at each point of $X_{1}(\mathbb{R})$ and $X_{2}(\mathbb{R})$ respectively. Such a map is called a birational diffeomorphism. In general a manifold can admit plenty of different models. For example, the hypersurfaces of $\mathbb{P}_{\mathbb{R}}^{3}$ given by the equations $x^{2 n}+y^{2 n}+z^{2 n}-t^{2 n}=0, n \geq 1$, provide infinitely many models of the sphere $\mathbb{S}^{2}$ which are pairwise not birational. Nevertheless, if one restricts to the simplest ones, namely the rational models, then for smooth compact manifolds of dimension at most 2 , the model is then unique. In dimension 1, we obtain only $\mathbb{P}^{1}$ and in dimension 2 this is the following result of Biswas and Huisman:

Theorem. [1, Corollary 8.1] A compact connected real manifold of dimension 2 admits a rational model if and only if it is non-orientable or diffeomorphic to $\mathbb{S}^{2}$ or $\mathbb{S}^{1} \times \mathbb{S}^{1}$. Moreover, this model is unique, up to birational diffeomorphism.

In the non-compact case, the real locus of the real affine algebraic variety $\mathbb{A}_{\mathbb{R}}^{2}$ provides an obvious rational algebraic model of the Euclidean plane $\mathbb{R}^{2}$ endowed with its standard structure of differential manifold. It is easy to find plenty of other rational models of $\mathbb{R}^{2}$ : we can choose for instance the complement in $\mathbb{P}_{\mathbb{R}}^{2}$ of a smooth irreducible real curve $\Gamma \subseteq \mathbb{P}_{\mathbb{R}}^{2}$ of odd degree $d \geq 3$ such that $\Gamma(\mathbb{R})$ is an oval equivalent to a line by a diffeomorphism of $\mathbb{R P}^{2}$. It is thus natural to restrict the study of such models to the smaller class of "Fake real planes", introduced in [5] as being smooth algebraic surfaces $S$ defined over $\mathbb{R}$, non isomorphic to $\mathbb{A}_{\mathbb{R}}^{2}$ but whose real locus is diffeomorphic to $\mathbb{R}^{2}$ and whose complexifications $S_{\mathbb{C}}$ have "minimal topology" in the sense that they are $\mathbb{Q}$-acyclic topological manifolds, that is, topological manifolds whose singular homology groups with rational coefficients $\tilde{H}_{i}\left(S_{\mathbb{C}} ; \mathbb{Q}\right)$ are all trivial.

By general results $[7,9,10,5]$ all these surfaces are affine and rational. A partial classification of them as real algebraic varieties was given in [5], according to their usual Kodaira dimension. Families of fake real planes of each Kodaira dimension $\kappa \in\{-\infty, 0,1,2\}$ birationally diffeomorphic to $\mathbb{A}_{\mathbb{R}}^{2}$ were constructed in [6]. The existence of fake real planes non birationally diffeomorphic to $\mathbb{A}_{\mathbb{R}}^{2}$ was left open.

Here we show that $\mathbb{R}^{2}$ admits algebraic models non birationally diffeomorphic to $\mathbb{A}_{\mathbb{R}}^{2}$ of every Kodaira dimension $\kappa=0,1,2$, answering the main question of [5] :

Theorem 1. There are infinitely many rational models $S$ of the plane $\mathbb{R}^{2}$ up to birational diffeomorphism, all having trivial reduced homology groups $\tilde{H}_{i}\left(S_{\mathbb{C}} ; \mathbb{Q}\right)$. Such models exist for every $\kappa=0,1,2$, and moreover, for $\kappa=1,2$, there exist infinitely many models $S$ up to birational diffeomorphism for which $S_{\mathbb{C}}$ is even topologically contractible.

In order to prove this result, we define a notion of real Kodaira dimension $\kappa_{\mathbb{R}}(S)$ (Definition 2.4), which has the property to be smaller than or equal to the classical one $\kappa(S)$, and can be computed in a very 
similar way (see Definition 2.4 and Remark 2.5). Moreover, we have equality $\kappa(S)=\kappa_{\mathbb{R}}(S)$ in the natural case where $S$ admits a smooth projective completion $V$ with SNC boundary $B=V \backslash S$ consisting only of real curves isomorphic to $\mathbb{P}_{\mathbb{R}}^{1}$, and intersecting only at real points. The main new noteworthy feature of $\kappa_{\mathbb{R}}(S)$ is that it is invariant under birational diffeomorphisms (Corollary 2.12), contrary to $\mathcal{K}(S)$ (Example 2.6).

We establish the following result, from which Theorem 1 directly follows.

Theorem 2. For each $l \in\{0,1,2\}$, there is a smooth affine surface $S$, algebraic model of the plane $\mathbb{R}^{2}$, with trivial rational homology groups $\tilde{H}_{i}\left(S_{\mathbb{C}} ; \mathbb{Q}\right)$ and $\kappa_{\mathbb{R}}(S)=\kappa(S)=l$. Moreover, for $l \in\{1,2\}$, we can find infinitely many such $S$ with topologically contractible complexifications $S_{\mathbb{C}}$, up to birational diffeomorphism.

As $\kappa_{\mathbb{R}}$ is invariant under birational diffeomorphisms, every fake real plane $S$ birationally diffeomorphic to $\mathbb{A}_{\mathbb{R}}^{2}$ satisfies $\kappa_{\mathbb{R}}(S)=-\infty$ (Corollary 2.15), so every of the examples which we construct in Theorem 2 is a fake real plane not birationally diffeomorphic to $\mathbb{A}_{\mathbb{R}}^{2}$.

In contrast with the cases $\kappa=1,2$, the only smooth algebraic models of $\mathbb{R}^{2}$ of Kodaira dimension $-\infty$ and 0 with $\mathbb{Q}$-acyclic complexifications known so far are respectively the affine plane $\mathbb{A}_{\mathbb{R}}^{2}$ and a real model $Y(3,3,3)$ of one of Fujita's exceptional surfaces [7] which was constructed in [5]. This motivates the following question:

Question 0.1. Are the surfaces $\mathbb{A}_{\mathbb{R}}^{2}$ and the fake real plane $Y(3,3,3)$ of real Kodaira dimension 0 given in $\S 2$. B.b the unique algebraic models of $\mathbb{R}^{2}$ with trivial reduced rational homology groups of real Kodaira dimension $-\infty$ and 0 , up to birational diffeomorphism?

The article is organised as follows: Section 1 contains some preliminaries. In Section 2, we define the real Kodaira dimension of a smooth real surface and establish its basic properties. We also give some examples of fake real planes of real Kodaira dimension 0 (the surface $Y(3,3,3)$ in $\S 2 . B . b$ ) and 2 (the Ramanujam surface in §2.B.b). Then, in Sections 3 and 4, we provide families of pairwise not birational diffeomorphic fake real planes of Kodaira dimension 1 and 2 respectively, which achieve the proof of Theorem 2 hence of Theorem 1. The last subsection $(\S 4 . C)$ describes pairs of fake real planes having the same complexifications but such that one has real Kodaira dimension 2 and the other is birationally diffeomorphic to $\mathbb{A}_{\mathbb{R}}^{2}$.

We thank the referee for his careful reading and his helpful comments to improve the exposition of this text.

\section{Preliminaries}

A k-variety is a geometrically integral scheme $X$ of finite type over a base field $\mathbf{k}$. A morphism of $\mathbf{k}$ varieties is a morphism of $\mathbf{k}$-schemes. In the sequel, $\mathbf{k}$ will be equal to either $\mathbb{R}$ or $\mathbb{C}$, and we will say that $X$ is a real, respectively complex, algebraic variety. A complex algebraic variety $X$ will be said to be defined over $\mathbb{R}$ if there exists a real algebraic variety $X_{0}$ and an isomorphism of complex algebraic varieties between $X$ and the complexification $X_{0, \mathbb{C}}=X_{0} \times_{\operatorname{Spec}(\mathbb{R})} \operatorname{Spec}(\mathbb{C})$ of $X_{0}$, where $\operatorname{Spec}(\mathbb{C}) \rightarrow \operatorname{Spec}(\mathbb{R})$ is the morphism induced by the usual inclusion $\mathbb{R} \hookrightarrow \mathbb{C}=\mathbb{R}[x] /\left(x^{2}+1\right)$.

\section{A. Real algebraic varieties and morphisms between them}

For a real algebraic variety $X$, we denote by $X(\mathbb{R})$ and $X(\mathbb{C})$ the sets of $\mathbb{R}$-rational and $\mathbb{C}$-rational points of $X$ respectively. These are endowed in a natural way with the Euclidean topology, locally induced by the usual Euclidean topologies on $\mathbb{A}_{\mathbb{R}}^{n}(\mathbb{R}) \simeq \mathbb{R}^{2 n}$ and $\mathbb{A}_{\mathbb{C}}^{n}(\mathbb{C}) \simeq \mathbb{C}^{n}$ respectively. When $X$ is smooth, $X(\mathbb{R})$ and $X(\mathbb{C})$ can be further equipped with natural structures of $\mathcal{C}^{\infty}$-manifolds. Every morphism $f: X \rightarrow X^{\prime}$ of real algebraic varieties induces a continuous map $X(\mathbb{R}) \rightarrow X^{\prime}(\mathbb{R})$ for the Euclidean topologies, and an isomorphism of real algebraic varieties $f: X \stackrel{\simeq}{\longrightarrow} X^{\prime}$ induces a homeomorphism $X(\mathbb{R}) \stackrel{\simeq}{\longrightarrow} X^{\prime}(\mathbb{R})$, which is a diffeomorphism when $X$ and $X^{\prime}$ are both smooth. 
In the context of the study of real algebraic models of a $\mathcal{C}^{\infty}$-manifold, it is natural to consider a broader class of isomorphisms, induced by appropriate rational maps. Recall that the domain of definition of a rational map $\varphi: X \rightarrow Y$ between two $\mathbf{k}$-schemes $X$ and $Y$ is the largest open subset $\operatorname{dom}_{\varphi}$ on which $\varphi$ is represented by a morphism. We say that $\varphi$ is regular at a closed point $x$ if $x \in \operatorname{dom}_{\varphi}$. A rational map $\varphi: X \rightarrow Y$ is called birational if it admits a rational inverse $\psi: Y \rightarrow X$.

Definition 1.1. Let $\varphi: X \rightarrow X^{\prime}$ be a rational map between real algebraic varieties such that $X(\mathbb{R})$ and $X^{\prime}(\mathbb{R})$ are not empty.

(1) We say that $\varphi$ is $\mathbb{R}$-regular, or that $\varphi$ induces a morphism $X(\mathbb{R}) \rightarrow X^{\prime}(\mathbb{R})$ (that we will again write $\varphi$ ), if the rational map $\varphi$ is regular at every $\mathbb{R}$-rational point of $X$. Equivalently, the real locus $X(\mathbb{R})$ of $X$ is contained in the domain of definition of $\varphi$.

(2) We say that $\varphi$ is $\mathbb{R}$-biregular, or that $\varphi$ is an isomorphism $X(\mathbb{R}) \stackrel{\simeq}{\longrightarrow} X^{\prime}(\mathbb{R})$, if it is birational and $\varphi$ and its inverse are $\mathbb{R}$-regular.

(3) A birational diffeomorphism is an $\mathbb{R}$-biregular rational map $\varphi: X \rightarrow X^{\prime}$ between smooth real algebraic varieties (or equivalently an isomorphism $X(\mathbb{R}) \stackrel{\simeq}{\longrightarrow} X^{\prime}(\mathbb{R})$, where $X$ and $X^{\prime}$ are smooth).

We can then consider the category most often used in real algebraic geometry (for instance in [1, 13, 2]) whose objects are the non-empty real loci $X(\mathbb{R})$ of real algebraic varieties and whose morphisms correspond to $\mathbb{R}$-regular rational maps $X(\mathbb{R}) \rightarrow X^{\prime}(\mathbb{R})$. Note that the class of morphisms considered is in general much larger than the class of usual regular maps. For instance, if $X$ is a projective real algebraic surface, the group $\operatorname{Aut}(X)$ of biregular automorphisms of $X$ is often quite small: its neutral component is an algebraic group and has thus finite dimension. In contrast, the group of birational diffeomorphisms $\operatorname{Aut}(X(\mathbb{R}))$ can be very large. If $X$ is smooth and rational, then $\operatorname{Aut}(X(\mathbb{R}))$ acts infinitely transitively on $X(\mathbb{R})[13$, Theorem 1.4]. A similar behaviour can also happen if $X$ is not rational but only geometrically rational [2, Theorem 2].

\section{B. Pairs and (logarithmic) Kodaira dimension}

Recall that a Smooth Normal Crossing $(S N C)$ divisor B on a smooth surface $S$ defined over $\mathbf{k}$ is a curve $B$ on $S$ whose base extension $B_{\overline{\mathbf{k}}}$ to the algebraic closure $\overline{\mathbf{k}}$ of $\mathbf{k}$ has smooth irreducible components and ordinary double points only as singularities. Equivalently, for every closed point $p \in B_{\overline{\mathbf{k}}} \subseteq S_{\overline{\mathbf{k}}}$, the local equations of the irreducible components of $B_{\overline{\mathbf{k}}}$ passing through $p$ form a part of a regular sequence in the maximal ideal $\mathrm{m}_{S_{\overline{\mathbf{k}}}, p}$ of the local ring $\mathcal{O}_{S_{\overline{\mathbf{k}}}, p}$ of $S_{\overline{\mathbf{k}}}$ at $p$.

Definition 1.2. A smooth $S N C$ pair $(V, B)$ is a pair consisting of a smooth projective surface $V$ and an SNC divisor $B \subseteq V$ both defined over $\mathbf{k}$.

By virtue of Nagata compactification $[19,20]$ and of classical desingularization theorems, every smooth surface $S$ defined over $\mathbf{k}$ admits an open embedding $S \hookrightarrow(V, B)$ into a smooth complete (in fact, projective by virtue of Chow Lemma) surface with possibly empty reduced SNC boundary divisor $B=V \backslash S$, both defined over $\mathbf{k}$. Such a pair $(V, B)$ is called a smooth $S N C$ completion of $S$.

The (logarithmic) Kodaira dimension $\kappa(S)$ of $S$ is then defined as the Iitaka dimension $\kappa\left(V, \omega_{V}(\log B)\right)$ [14], where

$$
\omega_{V}(\log B)=\left(\operatorname{det} \Omega_{V / \mathbf{k}}^{1}\right) \otimes \mathcal{O}_{V}(B) \simeq \mathcal{O}_{V}\left(K_{V}+B\right),
$$

for any canonical divisor $K_{V}$ on $V$. More explicitly, letting $R(V, B)=\bigoplus_{m \geq 0} H^{0}\left(V, \omega_{V}(\log B)^{\otimes m}\right)$ be the $\log$ canonical ring of the smooth SNC pair $(V, B)$, we have $\kappa(S)=\operatorname{tr} \operatorname{deg}_{\mathbf{k}} \mathcal{R}(V, B)-1$ if $H^{0}\left(V, \omega_{V}(\log B)^{\otimes m}\right) \neq 0$ for sufficiently large $m$ and otherwise, if $H^{0}\left(V, \omega_{V}(\log B)^{\otimes m}\right)=0$ for every $m \geq 1$, then we set by convention $\mathcal{K}(S)=-\infty$ and we say for short that $\kappa(S)$ is negative. The so-defined element $\mathcal{K}(S) \in\{-\infty, 0,1,2\}$ 
is independent of the choice of a smooth SNC completion $(V, B)$ of $S$ [15], and it coincides with the usual notion of Kodaira dimension in the case where $S$ is already complete. Furthermore, it is invariant under arbitrary extensions of the base field $\mathbf{k}$, as a consequence of the flat base change theorem [11, Proposition III.9.3]. In particular a smooth real surface $S$ and its complexification $S_{\mathbb{C}}=S \times_{\operatorname{Spec}(\mathbb{R})} \operatorname{Spec}(\mathbb{C})$ have the same Kodaira dimension.

\section{The real Kodaira dimension of open real surfaces}

\section{A. A variant of logarithmic Kodaira dimension}

For a smooth real surface $S$, the Kodaira dimension $\kappa(S)$ is in general not a birational invariant, unless $S$ is complete: for instance, the affine plane $\mathbb{A}_{\mathbb{R}}^{2}$ and the product of the punctured affine line $\mathbb{A}_{\mathbb{R}}^{1} \backslash\{0\}$ with itself are birational to each other but have Kodaira dimensions $-\infty$ and 0 respectively. We now introduce a variant of Kodaira dimension more adapted to the study of equivalence classes of open real surfaces up to birational diffeomorphisms.

Notation 2.1. Given a smooth SNC pair $(V, B)$ defined over $\mathbb{R}$, we denote by $B_{\mathbb{R}} \subseteq B$ the union of all irreducible components $B_{i}$ of $B_{\mathbb{C}}$ which are defined over $\mathbb{R}$ and such that $B_{i}(\mathbb{R})$ is infinite.

Remark 2.2. The Zariski closure of $B(\mathbb{R})$ in $V$ is the union of $B_{\mathbb{R}}$ and of finitely many isolated points of $B$.

Definition 2.3. Let $(V, B)$ be a smooth $\mathrm{SNC}$ pair defined over $\mathbb{R}$. We say that $B_{\mathbb{R}}$ contains an imaginary loop if there exists a pair of distinct irreducible components $A$ and $A^{\prime}$ of $B_{\mathbb{C}}$ defined over $\mathbb{R}$ and with infinite real loci, whose intersection $A \cap A^{\prime}$ contains a pair of conjugate non-real points, i.e. a $\mathbb{C}$-rational but not $\mathbb{R}$-rational point.

Definition 2.4. The real Kodaira dimension of a smooth SNC pair $(V, B)$ defined over $\mathbb{R}$ is the element

$$
\kappa_{\mathbb{R}}(V, B)=\kappa\left(V, \omega_{V}\left(\log B_{\mathbb{R}}\right)\right) \in\{-\infty, 0,1,2\} .
$$

If furthermore $B_{\mathbb{R}}$ has no imaginary loop, then we define the real Kodaira dimension of $S=V \backslash B$ to be

$$
\kappa_{\mathbb{R}}(S)=\kappa(S(\mathbb{R}))=\kappa_{\mathbb{R}}(V, B) .
$$

By definition, given a smooth SNC pair $(V, B)$ defined over $\mathbb{R}$, the curve $B_{\mathbb{R}}$ contains imaginary loops if and only if it has some pairs of non-real singular points $q$ and $\bar{q}$. The following lemma provides a simple procedure to eliminate imaginary loops.

Lemma 2.5. Let $(V, B)$ be a smooth $S N C$ pair defined over $\mathbb{R}$ and let $Z=\left\{q_{1}, \bar{q}_{1}, \ldots, q_{s}, \bar{q}_{s}\right\}$ be the set of non-real singular points of $B_{\mathbb{R}}$. Let $\tau: \hat{V} \rightarrow V$ be the blow-up of $Z$ and let $E=\sum_{i=1}^{s} \tau^{-1}\left(q_{i}\right)+\tau^{-1}\left(\bar{q}_{i}\right)$ be its exceptional locus. Then the following hold:

(1) $\left(\hat{V}, \hat{B}=\tau^{*}(B)_{\text {red }}\right)$ is a smooth $S N C$ pair defined over $\mathbb{R}$ for which $\hat{B}_{\mathbb{R}}$ has no imaginary loops and such that $\tau$ induces an isomorphism $\hat{V} \backslash \hat{B} \rightarrow V \backslash B$.

(2) $\kappa(\hat{V}, \hat{B})=\kappa(V, B)$ and $\kappa_{\mathbb{R}}(\hat{V}, \hat{B}) \leq \kappa_{\mathbb{R}}(V, B)$.

Proof. (1): As $B$ is SNC, the morphism $\tau$ only blows-up ordinary double points of $B$, so $\hat{B}$ is again SNC. Every irreducible curve on $\hat{V}_{\mathbb{C}}$ contracted by $\tau$ is not defined over $\mathbb{R}$ and does not intersect its conjugate, so does not contain any real point. This implies that $\hat{B}_{\mathbb{R}}$ is the strict transform of $B_{\mathbb{R}}$. Every singular $\mathbb{C}$ rational point of $B_{\mathbb{R}}$ which was not real has been blown-up, and every singular $\mathbb{C}$-rational point of $\hat{B}_{\mathbb{R}}$ is an $\mathbb{R}$-rational point. Hence, $\hat{B}_{\mathbb{R}}$ has no imaginary loop. The fact that $\tau$ induces an isomorphism $\hat{V} \backslash \hat{B} \rightarrow V \backslash B$ 
follows from the fact that $\hat{B}=\tau^{*}(B)_{\text {red }}$ and that all points blown-up by $\tau$ and all exceptional divisors of $E$ are contained in $B$ and $\hat{B}$ respectively.

(2): Since the points blown-up by $\tau$ are ordinary double points of $B_{\mathbb{R}}$ hence of $B$, we have $\hat{B}=\tau^{*} B-E$ whereas $\hat{B}_{\mathbb{R}}=\tau^{*} B_{\mathbb{R}}-2 E$ because $E$ does not contain any real point. Denoting by $K_{\hat{V}}$ and $K_{V}$ the canonical divisors on $\hat{V}$ and $V$ respectively, we have the ramification formula $K_{\hat{V}}=\tau^{*} K_{V}+E$ for $\tau$. This yields the two equalities

$$
K_{\hat{V}}+\hat{B}=\tau^{*}\left(K_{V}+B\right) \text { and } K_{\hat{V}}+\hat{B}_{\mathbb{R}}=\tau^{*}\left(K_{V}+B_{\mathbb{R}}\right)-E .
$$

The first equality gives $\kappa(\hat{V}, \hat{B})=\kappa(V, B)$. The second equality gives $\kappa_{\mathbb{R}}(\hat{V}, \hat{B}) \leq \kappa_{\mathbb{R}}(V, B)$, since $E$ is effective.

The following example shows that the inequality of Lemma $2.5(2)$ can be strict.

Example 2.6. Take $V=\mathbb{P}_{\mathbb{R}}^{2}$ and $B=L+C$, where $L \simeq \mathbb{P}_{\mathbb{R}}^{1}$ is the line of equation $x=0$ and $C$ is the smooth conic of equation $x^{2}-y^{2}-z^{2}=0$. Then, $B_{\mathbb{R}}=B$ has imaginary loops, as the points $q=[0: 1: i]$, $\bar{q}=[0: 1:-i]$ are singular points of $B$. With the notation of Lemma 2.5 , the blow-up $\tau: \hat{V} \rightarrow \mathbb{P}_{\mathbb{R}}^{2}$ of these two points yields an SNC pair $\left(\hat{V}, \hat{B}=\tau^{*}(B)_{\text {red }}\right)$ such that $\hat{B}=\tilde{L}+\tilde{C}+E$, where $E=E_{q}+E_{\bar{q}}$ is the sum of the exceptional divisors over $q$ and $\bar{q}$ respectively, and where $\tilde{L}, \tilde{C}$ are the strict transforms of $L$ and $C$. We get $\hat{B}_{\mathbb{R}}=\tilde{L}+\tilde{C}$.

The canonical divisor of $V=\mathbb{P}_{\mathbb{R}}^{2}$ satisfies $K_{V}=-L-C$ so that $K_{V}+B_{\mathbb{R}}=0$. On the other hand, by the proof of Lemma $2.5(2)$, we have $K_{\hat{V}}+\hat{B}_{\mathbb{R}}=\tau^{*}\left(K_{V}+B_{\mathbb{R}}\right)-E=-E$. Hence $\kappa_{\mathbb{R}}(V, B)=0$ whereas $\kappa_{\mathbb{R}}(\hat{V}, \hat{B})=-\infty$.

The aim of this section is to show that the definition of $\kappa(S(\mathbb{R}))$ (or $\kappa_{\mathbb{R}}(S)$ ) only depends on the birationnal diffeomorphism class of $S(\mathbb{R})$, or equivalently of the real surface $S$, up to birational diffeomorphism.

The following notion is natural to compare two possible pairs, up to birational diffeomorphism.

Definition 2.7. Let $(V, B)$ and $\left(V^{\prime}, B^{\prime}\right)$ be two smooth SNC pairs defined over $\mathbb{R}$. A birational map of pairs $\varphi:(V, B) \rightarrow\left(V^{\prime}, B^{\prime}\right)$ is a birational map $V \rightarrow V^{\prime}$ defined over $\mathbb{R}$ inducing an isomorphism

$$
(V \backslash B)(\mathbb{R}) \stackrel{\simeq}{\longrightarrow}\left(V^{\prime} \backslash B^{\prime}\right)(\mathbb{R})
$$

(or equivalently inducing a birational diffeomorphism from $V \backslash B$ to $V^{\prime} \backslash B^{\prime}$ ).

Example 2.8. Let $(V, B)$ and $\left(V^{\prime}, B^{\prime}\right)$ be two smooth SNC pairs defined over $\mathbb{R}$, and let $\tau: V \rightarrow V^{\prime}$ be a birational morphism, defined over $\mathbb{R}$. In each of the following cases, $\tau$ yields a birational map of pairs $(V, B) \rightarrow\left(V^{\prime}, B^{\prime}\right)$.

(1) If $\tau$ is an isomorphism $V \stackrel{\simeq}{\longrightarrow} V^{\prime}$ such that $\varphi(B(\mathbb{R}))=B^{\prime}(\mathbb{R})$.

(2) If $\tau$ is the blow-up of a point $q \in B^{\prime}(\mathbb{R})$ and $B=\varphi^{-1}\left(B^{\prime}\right)_{\text {red. }}$.

(3) If $\tau$ is the blow-up of a pair of conjugate non-real points $q, \bar{q} \in V^{\prime}(\mathbb{C})$ and $B$ is the strict transform of $B^{\prime}$. (Here the exceptional locus does not contain any real point.)

Remark 2.9. Another example of simple birational map of pairs $\tau:(V, B) \rightarrow\left(V^{\prime}, B^{\prime}\right)$ is given as follows: we take $\tau$ to be the blow-up of a pair of conjugate non-real points $q, \bar{q} \in V^{\prime}(\mathbb{C})$ and $B=\varphi^{-1}\left(B^{\prime}\right)_{\text {red }}$.

Denoting by $E \subseteq V$ the exceptional locus of $\tau$ (which is the disjoint union of two conjugate imaginary $(-1)$-curves and does not contain any real point) and by $\tilde{B}$ the strict transform of $B^{\prime}$, we get $B=\tilde{B}+E$. We can then decompose the birational map $\tau:(V, B) \rightarrow\left(V^{\prime}, B^{\prime}\right)$ as the composition of $\operatorname{id}_{V}:(V, B) \rightarrow(V, \tilde{B})$ with the birational morphism $\tau:(V, \tilde{B}) \rightarrow\left(V^{\prime}, B^{\prime}\right)$, which are examples of type (1) and (3) respectively. 
Lemma 2.10. Let $\varphi:(V, B) \rightarrow\left(V^{\prime}, B^{\prime}\right)$ be a birational map of smooth $S N C$ pairs. Then, there exists a sequence of birational maps of pairs

$$
(V, B)=\left(V_{0}, B_{0}\right) \stackrel{\varphi_{1}}{\rightarrow}\left(V_{1}, B_{1}\right) \stackrel{\varphi_{2}}{\rightarrow} \ldots \stackrel{\varphi_{n-1}}{\rightarrow}\left(V_{n-1}, B_{n-1}\right) \stackrel{\varphi_{n}}{\rightarrow}\left(V_{n}, B_{n}\right)=\left(V^{\prime}, B^{\prime}\right)
$$

such that $\varphi=\varphi_{n} \circ \cdots \circ \varphi_{1}$ and such that for each $i \in\{1, \ldots, n\}$, either $\varphi_{i}$ or $\left(\varphi_{i}\right)^{-1}$ is of one of three types (1)-(2)-(3) of Example 2.8.

Moreover, if $B_{\mathbb{R}}$ and $B_{\mathbb{R}}^{\prime}$ have no imaginary loop, then we can assume the same for $\left(B_{i}\right)_{\mathbb{R}}$, for $i=1, \ldots, n$.

Proof. By definition, $\varphi: V \rightarrow V^{\prime}$ is a birational map defined over $\mathbb{R}$, inducing an isomorphism between $(V \backslash B)(\mathbb{R})$ and $\left(V^{\prime} \backslash B^{\prime}\right)(\mathbb{R})$.

If $\varphi$ is an isomorphism $V \stackrel{\simeq}{\longrightarrow} V^{\prime}$, then it sends $B(\mathbb{R})$ onto $B^{\prime}(\mathbb{R})$ and is thus of the type of Example 2.8 (1). Otherwise, we can take a minimal resolution of the indeterminacies of $\varphi$ given by

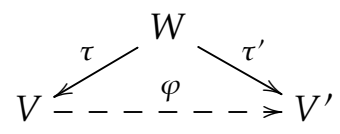

where $W$ is a smooth projective real surface and $\tau$ and $\tau^{\prime}$ are birational morphisms defined over $\mathbb{R}$. Recall that since $\varphi$ and $\varphi^{-1}$ are defined over $\mathbb{R}$, the union of their base-points, including infinitely near ones, is defined over $\mathbb{R}$, hence consists of either real points or pair of conjugate non-real points. The minimality assumption implies in particular that $\tau$ and $\tau^{\prime}$ are the blow-ups of the base-points of $\varphi$ and $\varphi^{-1}$ respectively. This gives back the classical decomposition of $\tau$ and $\tau^{\prime}$ into simple blow-ups (one real or a pair of conjugate non-real points) and thus the real Zariski strong factorisation of $\varphi$, as explained for instance in [22, Chapter II, Proposition 6.4].

We proceed by induction on the number of such points, the case where there is no base-point being $2.8(1)$.

If $q \in V(\mathbb{R})$ is a base-point of $\varphi$, then $q$ belongs to $B(\mathbb{R})$, since $\varphi$ induces an isomorphism between $(V \backslash B)(\mathbb{R})$ and $\left(V^{\prime} \backslash B^{\prime}\right)(\mathbb{R})$. We can write $\tau$ as $\tau=\tau_{q} \circ \hat{\tau}$, where $\tau_{q}: \hat{V} \rightarrow V$ is the blow-up of $q$ and $\hat{\tau}: W \rightarrow \hat{V}$ is a birational morphism defined over $\mathbb{R}$. Writing $\hat{B}=\left(\tau_{q}\right)^{-1}(B)_{\text {red }}$, the birational map $\tau_{q}$ yields a birational maps of pairs $(\hat{V}, \hat{B}) \rightarrow(V, B)$ of type $2.8(2)$. Moreover, if $B_{\mathbb{R}}$ has no imaginary loop, the same holds for $\hat{B}_{\mathbb{R}}=\left(\left(\tau_{q}\right)^{-1}\left(B_{\mathbb{R}}\right)\right)_{\text {red }}$. As $\varphi \circ \tau_{q}:(\hat{V}, \hat{B}) \rightarrow\left(V^{\prime}, B^{\prime}\right)$ is again a birational maps of pairs, whose minimal resolution has less base-points, we conclude by induction.

The same argument works with a point $q \in V^{\prime}(\mathbb{R})$ which is a base-point of $\varphi^{-1}$. We can thus assume that no point of $V(\mathbb{R})$ or $V^{\prime}(\mathbb{R})$ is a base-point of $\varphi$ or $\varphi^{-1}$.

If $\tau$ is not an isomorphism, there is a pair of conjugate non-real points $q, \bar{q} \in V(\mathbb{C})$, both base-points of $\varphi$, blown-up by $\tau$. As before, we write $\tau$ as $\tau=\tau_{q} \circ \hat{\tau}$, where $\tau_{q}: \hat{V} \rightarrow V$ is the blow-up of $q$ and $\bar{q}$, which is thus defined over $\mathbb{R}$. Then $\hat{\tau}: W \rightarrow \hat{V}$ is a birational morphism defined over $\mathbb{R}$. The strict transform $\hat{B}$ of $B$ on $\hat{V}$ is then again an SNC-divisor, defined over $\mathbb{R}$, and $\tau_{q}$ induces a birational map of pairs $(\hat{V}, \hat{B}) \rightarrow(V, B)$ of type $2.8(3)$. Moreover, if $B_{\mathbb{R}}$ has no imaginary loop, the same holds for $\hat{B}_{\mathbb{R}}$, which is the strict transform of $B_{\mathbb{R}}$. As before, the result follows by induction. The same works when $\tau^{\prime}$ is not a regular morphism.

Lemma 2.11. Let $\varphi:(V, B) \rightarrow\left(V^{\prime}, B^{\prime}\right)$ be a birational map of smooth $S N C$ pairs $(V, B)$ and $\left(V^{\prime}, B^{\prime}\right)$ defined over $\mathbb{R}$, such that neither $B_{\mathbb{R}}$ nor $B_{\mathbb{R}}^{\prime}$ has an imaginary loop. Then for every $m, n \in \mathbb{Z}$ with $m \geq|n|$, the map $\varphi$ induces an isomorphism

$$
\varphi_{*}: H^{0}\left(V, m K_{V}+n B_{\mathbb{R}}\right) \stackrel{\simeq}{\longrightarrow} H^{0}\left(V^{\prime}, m K_{V^{\prime}}+n B_{\mathbb{R}}^{\prime}\right) .
$$

In particular, $\kappa_{\mathbb{R}}(V, B)=\kappa_{\mathbb{R}}\left(V^{\prime}, B^{\prime}\right)$.

Proof. Applying Lemma 2.10, we can assume that $\varphi$ is of one of the three cases (1)-(2)-(3) of Example 2.8. 
In case (1), $\varphi$ is an isomorphism $V \stackrel{\simeq}{\longrightarrow} V^{\prime}$ that sends $B(\mathbb{R})$ isomorphically onto $B^{\prime}(\mathbb{R})$. It thus maps the Zariski closure of $B(\mathbb{R})$ isomorphically onto that of $B^{\prime}(\mathbb{R})$. This implies that $\varphi\left(B_{\mathbb{R}}\right)=B_{\mathbb{R}}^{\prime}$ (see Remark 2.2). This achieves the proof in this case.

We then do the two cases (2)-(3), and denote, in both cases, by $E \subseteq V$ the divisor contracted by $\varphi$.

In case (2), $\varphi$ is the blow-up of a point $q \in B^{\prime}(\mathbb{R}), B=\varphi^{-1}\left(B^{\prime}\right)_{\text {red }}$ and $E=\varphi^{-1}(q)$.

In case (3), $\varphi$ is the blow-up of a pair of conjugate non-real points $q, \bar{q} \in V^{\prime}(\mathbb{C}), B$ is the strict transform of $B^{\prime}$ and $E=\varphi^{-1}(q)+\varphi^{-1}(\bar{q})$. As $B_{\mathbb{R}}^{\prime}$ is an SNC-divisor with no imaginary loop, the points $q, \bar{q}$ cannot be singular points of $B_{\mathbb{R}}^{\prime}$.

We find respectively

$$
\begin{aligned}
& (2): \quad B_{\mathbb{R}}= \begin{cases}\varphi^{*}\left(B_{\mathbb{R}}^{\prime}\right)+E & \text { if } q \in B^{\prime}(\mathbb{R}) \backslash B_{\mathbb{R}}^{\prime}(\mathbb{R}), \\
\varphi^{*}\left(B_{\mathbb{R}}^{\prime}\right) & \text { if } q \text { is a simple point of } B_{\mathbb{R}}^{\prime}, \\
\varphi^{*}\left(B_{\mathbb{R}}^{\prime}\right)-E & \text { if } q \text { is a double point of } B_{\mathbb{R}}^{\prime},\end{cases} \\
& (3): \quad B_{\mathbb{R}}= \begin{cases}\varphi^{*}\left(B_{\mathbb{R}}^{\prime}\right) & \text { if } q \notin B_{\mathbb{R}}^{\prime}(\mathbb{C}), \\
\varphi^{*}\left(B_{\mathbb{R}}^{\prime}\right)-E & \text { if } q \in B_{\mathbb{R}}^{\prime}(\mathbb{C}) .\end{cases}
\end{aligned}
$$

Since $K_{V}=\varphi^{*}\left(K_{V^{\prime}}\right)+E$, we obtain

$$
m K_{V}+n B_{\mathbb{R}}=m\left(\varphi^{*}\left(K_{V^{\prime}}\right)+E\right)+n\left(\varphi^{*}\left(B_{\mathbb{R}}^{\prime}\right)+\epsilon E\right)=\varphi^{*}\left(m K_{V^{\prime}}+n B_{\mathbb{R}}^{\prime}\right)+\delta E,
$$

where $\epsilon \in\{-1,0,1\}$ and $\delta=m+n \epsilon \geq m-|n| \geq 0$ as $m \geq|n|$ by hypothesis. As a consequence, the natural inclusion

$$
H^{0}\left(V^{\prime}, m K_{V^{\prime}}+n B_{\mathbb{R}}^{\prime}\right) \simeq H^{0}\left(V, \varphi^{*}\left(m K_{V^{\prime}}+n B_{\mathbb{R}}^{\prime}\right)\right) \hookrightarrow H^{0}\left(V, \varphi^{*}\left(m K_{V^{\prime}}+n B_{\mathbb{R}}^{\prime}\right)+\delta E\right)
$$

is a bijection.

Indeed, for each integer $r \geq 0$, an effective divisor $D$ equivalent to $\varphi^{*}\left(m K_{V^{\prime}}+n B_{\mathbb{R}}^{\prime}\right)+r E$ is equal to $\tilde{D}+r E$ for some effective divisor $\tilde{D}$ equivalent to $\varphi^{*}\left(m K_{V^{\prime}}+n B_{\mathbb{R}}^{\prime}\right)$. This is clear for $r=0$, and for $r>0$ we just compute $E \cdot D=r E^{2} \leq-r<0$ and obtain that $D-E$ is effective, which yields the result by induction.

The case where $m=n$ shows that $\kappa_{\mathbb{R}}\left(V^{\prime}, B^{\prime}\right)=\kappa_{\mathbb{R}}(V, B)$.

As a consequence of Lemma 2.11, we obtain:

Corollary 2.12. For a smooth real affine surface $S, \kappa_{\mathbb{R}}(V, B)$ is independent of the choice of a smooth SNC completion $(V, B)$ of $S$ defined over $\mathbb{R}$ and such that $B_{\mathbb{R}}$ does not have an imaginary loop. The real Kodaira dimension $\kappa_{\mathbb{R}}(S) \in\{-\infty, 0,1,2\}$ of $S$ introduced in Definition 2.4 is thus a well-defined invariant of $S(\mathbb{R})$.

The following result summarises immediate consequences of the definition and Lemma 2.11.

Proposition 2.13. The real Kodaira dimension $\kappa_{\mathbb{R}}(S)$ of a smooth real surface $S$ enjoys the following properties:

(1) $\kappa_{\mathbb{R}}(S)=\kappa_{\mathbb{R}}\left(S^{\prime}\right)$ for every smooth surface $S^{\prime}$ defined over $\mathbb{R}$ birationally diffeomorphic to $S$.

(2) $\kappa_{\mathbb{R}}(S) \leq \kappa(S)$ with equality if $S$ admits a smooth projective $S N C$ completion $(V, B)$ defined over $\mathbb{R}$ such that $B=B_{\mathbb{R}}$ has no imaginary loop.

Proof. Assertion (1) follows from Lemma 2.11. For (2), it follows from Lemma 2.5 that the surface $S$ always admits a smooth projective SNC completion $(V, B)$ defined over $\mathbb{R}$ such that $B_{\mathbb{R}}$ has no imaginary loop. We then have $B=B_{\mathbb{R}}+E$ for some effective divisor $E$, and thus get $\kappa_{\mathbb{R}}(S)=\kappa_{\mathbb{R}}(V, B)=\kappa\left(V, B_{\mathbb{R}}\right) \leq \kappa(V, B)=$ $\kappa(S)$, with equality if $B=B_{\mathbb{R}}$. 
Example 2.14. The inequality $\kappa_{\mathbb{R}}(S) \leq \kappa(S)$ is strict in general: for instance, let $B \subseteq \mathbb{P}_{\mathbb{R}}^{2}$ be a general arrangement consisting of $0 \leq r \leq 2$ real lines and a collection of $p \geq 0$ pairs of non-real complex conjugate lines. Then for $S=\mathbb{P}_{\mathbb{R}}^{2} \backslash B$, we have $\kappa_{\mathbb{R}}(S)=-\infty$ independently of $r$ and $p$ while

$$
\kappa(S)= \begin{cases}-\infty & \text { if } r+2 p<3, \\ 0 & \text { if } r+2 p=3, \\ 2 & \text { if } r+2 p \geq 4 .\end{cases}
$$

The equality $\kappa_{\mathbb{R}}(S)=-\infty$ follows from the fact that $S$ is birationally diffeomorphic to the complement $S^{\prime}$ of $r \leq 2$ lines in $\mathbb{P}_{\mathbb{R}}^{2}$, which satisfies $\kappa_{\mathbb{R}}\left(S^{\prime}\right)=\kappa(S)=-\infty$. On the other hand, since $B$ is an SNC divisor, $\kappa(S)=\kappa\left(\mathbb{P}_{\mathbb{R}}^{2}, B\right)$ where $K_{\mathbb{P}_{\mathbb{R}}^{2}}+B$ has degree $-3+r+2 p$.

As a consequence of Proposition 2.13 (1), we obtain:

Corollary 2.15. Let $S$ be a smooth real surface. If $S$ is birationally diffeomorphic to $\mathbb{A}_{\mathbb{R}}^{2}$, then $\kappa_{\mathbb{R}}(S)=-\infty$.

Proof. Follows from Proposition 2.13 (1), and the fact that $\mathbb{A}_{\mathbb{R}}^{2}=\mathbb{P}_{\mathbb{R}}^{2} \backslash L$, where $L \subseteq \mathbb{P}_{\mathbb{R}}^{2}$ is a real line. Hence, $m\left(K_{\mathbb{P}_{\mathbb{R}}^{2}}+L\right) \simeq-2 m L$ is not effective for each $m \geq 0$, so $\kappa_{\mathbb{R}}(S)=\kappa_{\mathbb{R}}\left(\mathbb{A}_{\mathbb{R}}^{2}\right)=\kappa\left(\mathbb{P}_{\mathbb{R}}^{2}, L\right)=-\infty$.

\section{B. Examples}

\section{B.a. An algebraic model of real Kodaira dimension 0: the exceptional fake plane $Y(3,3,3)$}

Let us recall from [5, §5.1.1] the following construction of a fake plane $S$ of Kodaira dimension 0 whose complexification $S_{\mathbb{C}}$ is $\mathbb{Q}$-acyclic ${ }^{1}$, with $H_{1}\left(S_{\mathbb{C}} ; \mathbb{Z}\right) \simeq \mathbb{Z}_{9}$. Let $D$ be the union of four general real lines $\ell_{i} \simeq \mathbb{P}_{\mathbb{R}}^{1}, i=0,1,2,3$ in $\mathbb{P}_{\mathbb{R}}^{2}$ and let $\tau: V \rightarrow \mathbb{P}_{\mathbb{R}}^{2}$ be the real projective surface obtained by first blowingup the real points $p_{i j}=\ell_{i} \cap \ell_{j}$ with exceptional divisors $E_{i j}, i, j=1,2,3, i \neq j$ and then blowing-up the real points $\ell_{1} \cap E_{12}, \ell_{2} \cap E_{23}$ and $\ell_{3} \cap E_{13}$ with respective exceptional divisors $E_{1}, E_{2}$ and $E_{3}$. We let $B=\ell_{0} \cup \ell_{1} \cup \ell_{2} \cup \ell_{3} \cup E_{12} \cup E_{23} \cup E_{13}$. The dual graphs of $D$, of its total transform $\tau^{-1}(D)$ in $V$ and of $B$ are depicted in Figure 1.

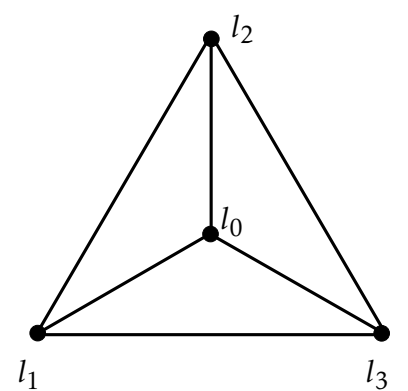

D

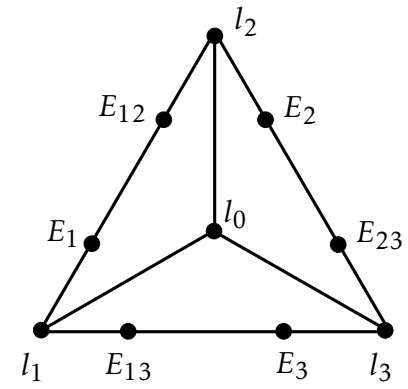

$\tau^{-1}(D)$

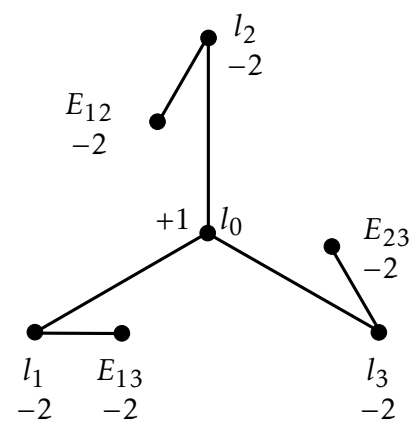

$B$

Figure 1: Construction of $Y(3,3,3)$

By virtue of $[5, \S 5.1 .1]$ (see also $[6, \S 3.2])$, the real surface $Y(3,3,3)=V \backslash B$ is a fake real plane of Kodaira dimension $\kappa(Y(3,3,3))=0$. Since by construction $B=B_{\mathbb{R}}$ is a tree, we conclude by Proposition $2.13(1)$ that $\kappa_{\mathbb{R}}(Y(3,3,3))=\kappa(Y(3,3,3))=0$, hence that $Y(3,3,3)$ is not birationally diffeomorphic to $\mathbb{A}_{\mathbb{R}}^{2}$. This answers [5, Question 5.2].

$1 \uparrow$ It is known that there is no fake real plane of Kodaira dimension 0 with $\mathbb{Z}$-acyclic complexification [17, Theorem 4.7.1(1), p. 244] 


\section{B.b. An algebraic model of real Kodaira dimension 2: the real Ramanujam surface}

The real Ramanujam surface $S$ is a real model of the complex Ramanujam surface [21] which is constructed as follows: let $D \subseteq \mathbb{P}_{\mathbb{R}}^{2}=\operatorname{Proj}(\mathbb{R}[x, y, z])$ be the union of the cuspidal cubic $C=\left\{x^{2} z+y^{3}=0\right\}$ with its osculating conic $Q$ at an $\mathbb{R}$-rational point $q \in C(\mathbb{R})$ distinct from the singular point $[0: 0: 1]$ of $C$ and its flex $[0: 0: 1]$. Up to change of coordinates, one can for instance choose $q=[1: 1:-1]$, which implies that the equation of $Q$ is

$$
5 x^{2}+24 x y-40 x z+45 y^{2}-15 y z-z^{2} .
$$

So $Q$ is a smooth $\mathbb{R}$-rational conic intersecting $C$ at $q$ with multiplicity 5 and transversally at a second $\mathbb{R}$-rational point $p$. We let $\beta: \mathbb{F}_{1} \rightarrow \mathbb{P}_{\mathbb{R}}^{2}$ be the blow-up of $p$ with exceptional divisor $E \simeq \mathbb{P}_{\mathbb{R}}^{1}$ and we let $S$ be the complement in $\mathbb{F}_{1}$ of the proper transform $\tilde{D}$ of $D$. The total transform $B$ of $\tilde{D}$ in a minimal $\log$-resolution $\tau:(V, B) \rightarrow\left(\mathbb{F}_{1}, \tilde{D}\right)$ of the pair $\left(\mathbb{F}_{1}, \tilde{D}\right)$ is a tree of $\mathbb{R}$-rational curves depicted in Figure 2 .

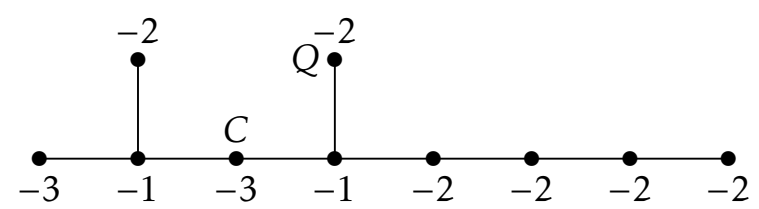

Figure 2: The weighted dual graph of the divisor $B \subseteq V$.

The surface $S$ is a fake real plane of Kodaira dimension 2 with contractible complexification $S_{\mathbb{C}}$ : the contractibility of $S_{\mathbb{C}}$ was first established by Ramanujam [21], the fact that $\kappa(S)=2$ follows for instance from the classification of contractible complex surfaces of Kodaira dimension $\leq 1$ established in [8] (see also [16]), and the fact that $S(\mathbb{R}) \simeq \mathbb{R}^{2}$ was proven in [5, Example 3.8].

Since the smooth SNC completion $(V, B)$ of $S$ has the property that $B_{\mathbb{R}}=B$ is a tree, the equality $\kappa_{\mathbb{R}}(S)=\kappa(S)=2$ holds by virtue of Proposition $2.13(1)$, and so, $S$ is not birationally diffeomorphic to $\mathbb{A}_{\mathbb{R}}^{2}$.

Remark 2.16. The same argument as above also applies to the three examples of fake real planes $S$ of $\log$ general type with contractible complexification $S_{\mathbb{C}}$ constructed in $[6, \S 5.1]$ from arrangements of real lines and irreducible singular $\mathbb{R}$-rational quartics in $\mathbb{P}_{\mathbb{R}}^{2}$ : all these surfaces have the property to admit a smooth SNC completion $(V, B)$ defined over $\mathbb{R}$ for which $B_{\mathbb{R}}=B$ is a tree, so that their real Kodaira dimension $\kappa_{\mathbb{R}}$ coincides with their usual Kodaira dimension. All of them are therefore non birationally diffeomorphic to $\mathbb{A}_{\mathbb{R}}^{2}$.

\section{Families of algebraic models of Kodaira dimension 1}

Fake real planes $S$ of Kodaira dimension 1 whose complexifications $S_{\mathbb{C}}$ are $\mathbb{Z}$-acyclic manifolds, that is topological manifolds with trivial reduced homology groups $\tilde{H}_{i}\left(S_{\mathbb{C}} ; \mathbb{Z}\right)$, have been classified up to isomorphism in [5] (see also [8] and [3] for the complex case). One obtains the following:

Proposition 3.1. A fake real plane of Kodaira dimension $\kappa=1$ with $\mathbb{Z}$-acyclic complexification is not birationally diffeomorphic to $\mathbb{A}_{\mathbb{R}}^{2}$.

Proof. By virtue of [5, Theorem 3.2], every such surface admits a completion into a smooth projective surface $V$ defined over $\mathbb{R}$ obtained from $\mathbb{P}_{\mathbb{R}}^{2}$ by blowing-up specific sequences of real points, and whose boundary $B=V \backslash S$ consists of a tree of $\mathbb{P}_{\mathbb{R}}^{1}$ 's. In particular, such a smooth pair $(V, B)$ satisfies $B_{\mathbb{R}}=B$, and we deduce from Proposition $2.13(1)$ that $\kappa_{\mathbb{R}}(S)=\kappa(S)=1$. The result then follows from Corollary 2.15.

In the rest of this section, we build on a blow-up construction of certain fake real planes of Kodaira dimension 1 with contractible complexifications [5, Example 3.5] to derive the existence of infinitely many pairwise non birationally diffeomorphic such surfaces. The main ingredient is the uniqueness of the $\log$ canonical fibration, given by Lemma 2.11 . 
Let $1<a<b$ be a pair of coprime integers and consider the rational pencil

$$
\Psi: \mathbb{P}_{\mathbb{R}}^{2}=\operatorname{Proj}_{\mathbb{R}}(\mathbb{R}[x, y, z]) \rightarrow \mathbb{P}_{\mathbb{R}}^{1}, \quad[x: y: z] \mapsto\left[y^{b}: x^{a} z^{b-a}\right] .
$$

It has two proper base points $q_{0}=[0: 0: 1]$ and $q_{\infty}=[1: 0: 0]$. A general geometrically irreducible fiber of $\Psi$ is an $\mathbb{R}$-rational cuspidal curve, with multiplicity $a$ and $b-a$ at $q_{0}$ and $q_{\infty}$ respectively, and $\Psi$ has precisely two degenerate members: $\Psi^{-1}([1: 0])$ which is supported on the union of the lines $L_{x}=\{x=0\}$ and $L_{z}=\{z=0\}$ and $\Psi^{-1}([0: 1])$ which is supported on the line $L_{y}=\{y=0\}$. Up to exchanging the roles of $x$ and $z$, we assume from now on that $a>b-a$.

Let $C_{a, b}=\Psi^{-1}([1:-1])=\left\{x^{a} z^{b-a}-y^{b}=0\right\}$, let $p=[1: 1: 1] \in C_{a, b}$ and let $\beta: X(a, b) \rightarrow \mathbb{P}_{\mathbb{R}}^{2}$ be the blow-up of $p$, with exceptional divisor $E$. We let $S(a, b)=X(a, b) \backslash\left(C_{a, b} \cup L_{z}\right)$ where we identified a curve in $\mathbb{P}_{\mathbb{R}}^{2}$ with its proper transform in $X(a, b)$.

The dual graph of the total transform of $C_{a, b} \cup L_{x} \cup L_{y} \cup L_{z}$ in the minimal resolution $\alpha: V(a, b) \rightarrow X(a, b)$ of the induced rational map $\Psi \circ \beta: X(a, b) \rightarrow \mathbb{P}_{\mathbb{R}}^{1}$ is depicted in Figure 3. The boundary $B(a, b)=V(a, b) \backslash$ $S(a, b)$ is the reduced total transform of $C_{a, b} \cup L_{z}$. The induced morphism $f=\Psi \circ \beta \circ \alpha: V(a, b) \rightarrow \mathbb{P}_{\mathbb{R}}^{1}$ is a $\mathbb{P}^{1}$-fibration having the last exceptional divisors $C_{0}$ and $C_{1}$ of $\alpha$ over the points $q_{0}$ and $q_{\infty}$ as disjoint sections.

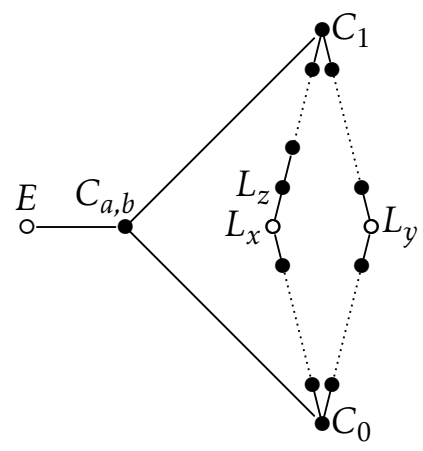

Figure 3: The dual graph of the total transform of $C_{a, b} \cup L_{x} \cup L_{y} \cup L_{z}$, the components denoted by $\circ$ are those which do not belong to the boundary $B(a, b)$.

Proposition 3.2. For every pair of coprime integers $1<a<b$, the surface $S(a, b)$ is a fake real plane of Kodaira dimension $\kappa=\kappa_{\mathbb{R}}=1$, with contractible complexification. Furthermore, if $(a, b) \neq\left(a^{\prime}, b^{\prime}\right)$ then $S(a, b)$ and $S\left(a^{\prime}, b^{\prime}\right)$ are not birationally diffeomorphic.

Proof. The first assertion follows from [5, Theorem 3.2] and Proposition 2.13 (1) using the fact that $B_{\mathbb{R}}(a, b)=$ $B(a, b)$ is a tree. Set $S=S(a, b)$ and $S^{\prime}=S\left(a^{\prime}, b^{\prime}\right)$ and suppose that there exists a birational diffeomorphism $\varphi: S \rightarrow S^{\prime}$. Let $(V, B)=(V(a, b), B(a, b))$ and $\left(V^{\prime}, B^{\prime}\right)=\left(V\left(a^{\prime}, b^{\prime}\right), B\left(a^{\prime}, b^{\prime}\right)\right)$ be the smooth pairs obtained by taking the minimal resolutions of the pencils $\Psi \circ \beta$ and $\Psi^{\prime} \circ \beta^{\prime}$ respectively. The structures of $B$ and $B^{\prime}$ imply that $\varphi$ extends to a birational diffeomorphism of pairs $\Phi:(V, B) \rightarrow\left(V^{\prime}, B^{\prime}\right)$. Indeed, otherwise either $\Phi$ or its inverse, say $\Phi$, would contract an irreducible component of $B$ onto a real point of $B^{\prime}$. But $B$ does not contain any irreducible curve whose proper transform by a birational morphism $W \rightarrow V$ defined over $\mathbb{R}$ whose center is supported on $B$ is $(-1)$-curve which can be contracted while keeping the property that the total transform of $B$ is an SNC divisor.

By virtue of [17, Lemma 4.5.3 p. 237], the positive part of the Zariski decomposition of $K_{V}+B$ is equal to $\left(1-\frac{1}{a}-\frac{1}{b}\right) \ell$ where $\ell$ denotes a general real fiber of the $\mathbb{P}^{1}$-fibration $f: V \rightarrow \mathbb{P}_{\mathbb{R}}^{1}$. Since $1<a<b$, it follows that $f$ coincides with the log-canonical fibration $f_{\left|m\left(K_{V}+B\right)\right|}: V \rightarrow \mathbb{P}_{\mathbb{R}}^{1}$ for every integer $m \geq 1$. The same holds for the log-canonical fibration $f^{\prime}=f_{\left|m\left(K_{V^{\prime}}+B^{\prime}\right)\right|}: V^{\prime} \rightarrow \mathbb{P}_{\mathbb{R}}^{1}$ on $V^{\prime}$. Since $B=B_{\mathbb{R}}$ and similarly for $B^{\prime}$, it follows from Lemma 2.11 that for every $m \geq 1$, $\Phi$ induces an isomorphism between $H^{0}\left(V, m\left(K_{V}+B\right)\right)$ and $H^{0}\left(V^{\prime}, m\left(K_{V^{\prime}}+B^{\prime}\right)\right)$. Consequently, there exists an automorphism $\gamma$ of $\mathbb{P}_{\mathbb{R}}^{1}$ defined over $\mathbb{R}$ such that $f^{\prime} \circ \Phi=\gamma \circ f$. 
The curves $E, L_{x}$ and $L_{y}$ have multiplicities $1, a$ and $b$ as irreducible components of the scheme theoretic fibers of $f: V \rightarrow \mathbb{P}_{\mathbb{R}}^{1}$ over the points $[1: 1]$, [1:0] and [0:1] respectively. Similarly, the curves $E^{\prime}, L_{x}^{\prime}$ and $L_{y}^{\prime}$ have multiplicities $1, a^{\prime}$ and $b^{\prime}$ as irreducible components of the scheme theoretic fibers of $f^{\prime}: V^{\prime} \rightarrow \mathbb{P}_{\mathbb{R}}^{1}$ over these points. Since $1<a<b$ and $1<a^{\prime}<b^{\prime}$, and $\Phi(S(\mathbb{R})) \subseteq S^{\prime}(\mathbb{R})$, it follows that $\Phi_{*}(E)=E^{\prime}, \Phi_{*}\left(L_{x}\right)=L_{x}^{\prime}$ and $\Phi_{*}\left(L_{y}\right)=L_{y}^{\prime}$. Thus $\gamma=\mathrm{id}$, from which we conclude in turn that $a=a^{\prime}$ and $b=b^{\prime}$.

\section{Families of algebraic models of Kodaira dimensions 2}

Here we construct infinite families of pairwise non birationally diffeomorphic fake real planes of real Kodaira dimension 2 with contractible complexifications. We also give examples of $\mathbb{Z}$-acyclic complex surfaces of log-general type with two real forms: one of them has negative logarithmic Kodaira real dimension and is in fact birationally diffeomorphic to $\mathbb{A}_{\mathbb{R}}^{2}$ whereas the other one has real Kodaira dimension 2, hence is not birationally diffeomorphic to $\mathbb{A}_{\mathbb{R}}^{2}$.

\section{A. A criterion for isomorphism}

Lemma 4.1. Let $S$ be a fake real plane of real Kodaira dimension 2, with $\mathbb{Q}$-acyclic complexification. Suppose that there exists a smooth $S N C$ completion $(V, B)$ of $S$ defined over $\mathbb{R}$ for which $B=B_{\mathbb{R}}$. Then the log-canonical rational map

$$
\varphi: V \rightarrow \operatorname{Proj}\left(\bigoplus_{m \geq 0} H^{0}\left(V, m\left(K_{V}+B_{\mathbb{R}}\right)\right)\right)
$$

is a morphism, which restricts to an isomorphism between $S$ and its image.

Proof. By hypothesis, $S_{\mathbb{C}}$ is a smooth $\mathbb{Q}$-acyclic surface of Kodaira dimension $\kappa\left(S_{\mathbb{C}}\right)=\kappa(S)=\kappa_{\mathbb{R}}(S)=2$. By the Bogomolov-Miyaoka-Yau inequality (see e.g. [17, Theorem 6.6.2]) $S_{\mathbb{C}}$ does not contain any topologically contractible algebraic curve. Since $S_{\mathbb{C}}$ is affine and rational, it follows from [17, Lemma 1.5.1 p. 198] that the only curves contracted by a $K_{V_{\mathbb{C}}}+B_{\mathbb{C}}$-MMP ran from $\left(V_{\mathbb{C}}, B_{\mathbb{C}}\right)$ are irreducible components of $B_{\mathbb{C}}$. The assumption that $B=B_{\mathbb{R}}$ implies that such a MMP is defined over $\mathbb{R}$. Let $h:(V, B) \rightarrow(W, \Delta)$ be the corresponding birational morphism, where $\Delta=h_{*} B$. Then $(W, \Delta)$ is an lc pair defined over $\mathbb{R}$, such that $K_{W}+\Delta$ is semi-ample [17, Theorem 4.12.1], and $h$ restricts to an isomorphism $S=V \backslash B \stackrel{\simeq}{\rightarrow} W \backslash \Delta$. We have $K_{V}+B=h^{*}\left(K_{W}+\Delta\right)+E$ where $E$ is an effective $\mathbb{Q}$-divisor supported on the exceptional locus of $h$, and $h$ induces an isomorphism $h^{*}: H^{0}\left(W, m\left(K_{W}+\Delta\right)\right) \stackrel{\sim}{\rightarrow} H^{0}\left(V, m\left(K_{V}+B\right)\right)=H^{0}\left(V, m\left(K_{V}+B_{\mathbb{R}}\right)\right)$ for every $m \geq 0$. Finally, again due to the fact that $S_{\mathbb{C}}$ is affine, rational and does not contain any topologically contractible algebraic curve, it follows from [17, Lemma 1.6.1 p. 200] that the only curves that could be contracted by the log-canonical morphism

$$
\psi: W \rightarrow \operatorname{Proj}\left(\bigoplus_{m \geq 0} H^{0}\left(W, m\left(K_{W}+\Delta\right)\right)\right) \simeq \operatorname{Proj}\left(\bigoplus_{m \geq 0} H^{0}\left(V, m\left(K_{V}+B_{\mathbb{R}}\right)\right)\right)
$$

are irreducible components of $\Delta$. So $\varphi=\psi \circ h$ restricts to an isomorphism between $S$ and its image.

By combining Lemma 2.11 and Lemma 4.1, we obtain the following:

Proposition 4.2. Let $S$ and $S^{\prime}$ be fake real planes of real Kodaira dimension 2 with $\mathbb{Q}$-acyclic complexifications. Assume further that there exist $S N C$ minimal completions $(V, B)$ and $\left(V^{\prime}, B^{\prime}\right)$ of $S$ and $S^{\prime}$ respectively defined over $\mathbb{R}$ such that $B=B_{\mathbb{R}}$ and $B^{\prime}=B_{\mathbb{R}}^{\prime}$. Then every birational diffeomorphism $f: S \rightarrow S^{\prime}$ is an isomorphism.

Proof. Let $F:(V, B) \rightarrow\left(V^{\prime}, B^{\prime}\right)$ be the birational map of pairs induced by $f$. The hypothesis implies that the boundaries $B_{\mathbb{R}}=B$ and $B_{\mathbb{R}}^{\prime}=B^{\prime}$ are trees of $\mathbb{R}$-rational curves [5, Lemma 2.3]. So by 2.11, $\varphi$ induces an 
isomorphism $\theta$ between the $\log$-canonical rings

$$
R\left(V, B_{\mathbb{R}}\right)=\bigoplus_{m \geq 0} H^{0}\left(V, m\left(K_{V}+B_{\mathbb{R}}\right)\right) \quad \text { and } \quad R\left(V^{\prime}, B_{\mathbb{R}}^{\prime}\right)=\bigoplus_{m \geq 0} H^{0}\left(V^{\prime}, m\left(K_{V^{\prime}}+B_{\mathbb{R}}^{\prime}\right)\right)
$$

of the pairs $\left(V, B_{\mathbb{R}}\right)$ and $\left(V^{\prime}, B_{\mathbb{R}}^{\prime}\right)$ respectively. On the other hand, it follows from Lemma 4.1 that the $\log$-canonical morphisms $\varphi: V \rightarrow X=\operatorname{Proj}\left(R\left(V, B_{\mathbb{R}}\right)\right)$ and $\varphi^{\prime}: V^{\prime} \rightarrow X^{\prime}=\operatorname{Proj}\left(R\left(V^{\prime}, B_{\mathbb{R}}^{\prime}\right)\right)$ restrict to isomorphisms $S=V \backslash B_{\mathbb{R}} \simeq X \backslash \varphi_{*}\left(B_{\mathbb{R}}\right)$ and $S^{\prime}=V^{\prime} \backslash B_{\mathbb{R}}^{\prime} \simeq X^{\prime} \backslash \varphi_{*}^{\prime}\left(B_{\mathbb{R}}^{\prime}\right)$. We thus get a commutative diagram

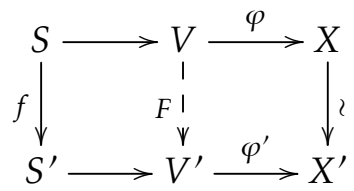

where the right-hand side isomorphism is induced by $\theta$. This shows that $f$ is an isomorphism.

\section{B. Miyanishi-Sugie surfaces: a countable family of pairwise non birationally diffeomet- ric fake real planes of log-general type}

We consider the following real counterpart of a family of smooth complex topologically contractible surfaces of $\log$-general type constructed by Miyanishi-Sugie [18]. For each integer $s \geq 1$, we will construct a surface $S_{s}$, defined over $\mathbb{R}$, which corresponds to the surface $X_{s+1,1}^{(1)}$ of [18], i.e. to the construction of [18] with $n=s+1, m=1$ and $r=s$. We recall the construction here for self-containedness.

We define $C_{s}$ and $L_{s}$ to be the irreducible curves in $\mathbb{P}_{\mathbb{R}}^{2}=\operatorname{Proj}(\mathbb{R}[x, y, z])$ given by the zero loci of the polynomials

$$
y^{s}\left(\left(s^{2}-1\right) x+s y-z\right)+(x-s y)(x+y)^{s} \text { and }\left(\left(s^{2}-1\right) x+s y-z\right)
$$

respectively. Note that the polynomials above correspond to $-y^{n-1} z+x^{2}\left(x^{n-2}-\sum_{i=2}^{n-2}(i-1)\left(\begin{array}{c}n \\ i\end{array}\right) x^{n-i-2} y^{i}\right)$ and $\left(n^{2}-2 n\right) x+(n-1) y-z$, with $n=s+1$, and thus the equations of $C_{s}$ and $L_{s}$ are the same as those given in [18, Lines 1-2, Page 338].

The curve $C_{s}$ is rational, of degree $s+1$ with a unique cuspidal singularity of multiplicity $s$ at the point $[0: 0: 1]$, which is solved by one blow-up. The line $L_{s}$ is the tangent line to $C_{s}$ at the point $p=\left[1:-1: s^{2}-s-1\right]$ and intersects $C_{s}$ with multiplicity $s$ at $p$ and with multiplicity 1 at the point $q=\left[s: 1: s^{3}\right]$.

Let $\tau: V_{0, s} \rightarrow \mathbb{P}_{\mathbb{R}}^{2}$ be the birational morphism defined over $\mathbb{R}$ obtained by first blowing-up $q$ and then blowing-up $s+1$ times the intersection point of the proper transform of $C_{s}$ with that of the previous exceptional divisor produced. Let $E_{1}, \ldots, E_{s+2} \simeq \mathbb{P}_{\mathbb{R}}^{1}$ be the corresponding successive exceptional divisors. Let $B_{0, s}=C_{s} \cup L_{s} \cup \bigcup_{i=1}^{s+1} E_{i}$, where we identified each curve with its proper transform in $V_{0, s}$ and let $S_{s}=V_{0, s} \backslash B_{0, s}$. The dual graph of $B_{0, s}$ is given in the following figure, where the double arrow corresponds to a multiple intersection at a point (corresponding here to the point $p$ ).

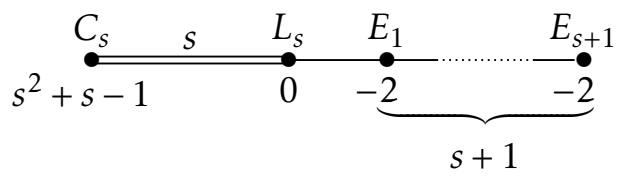

The smooth surface $S_{s}$ is defined over $\mathbb{R}$. A minimal log-resolution $f_{s}:\left(V_{s}, B_{s}\right) \rightarrow\left(V_{0, s}, B_{0, s}\right)$ defined over $\mathbb{R}$ of the pair $\left(V_{0, s}, B_{0, s}\right)$ is obtained by taking a log-resolution of the singular point $[0: 0: 1]$ of $C_{s}$ and blowing-up the real point $p$ and its infinitely near points $s$ times to separate the proper transforms of $C_{s}$ and $L_{s}$.

After blowing-up the singular point of $C_{s}$ on $V_{0, s}$, the total transform of $B_{0, s}$ is given by the following dual graph, where $F$ is the exceptional curve contracted on the singular point of $C_{s}$. 


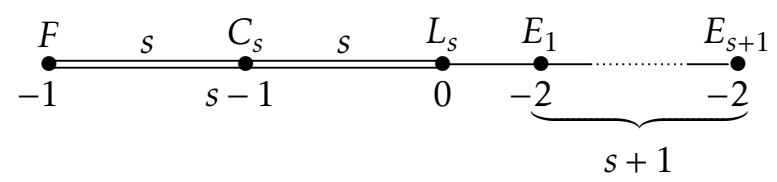

The total transform $B_{S}$ of $B_{0, s}$ is thus a tree of $\mathbb{R}$-rational curves whose dual graph is depicted on Figure 4 (which is the same as the graph of $X_{n, m}^{(1)}$ given in [18, Theorem 1, Page 339], with $m=1$ and $n=s+1)$.

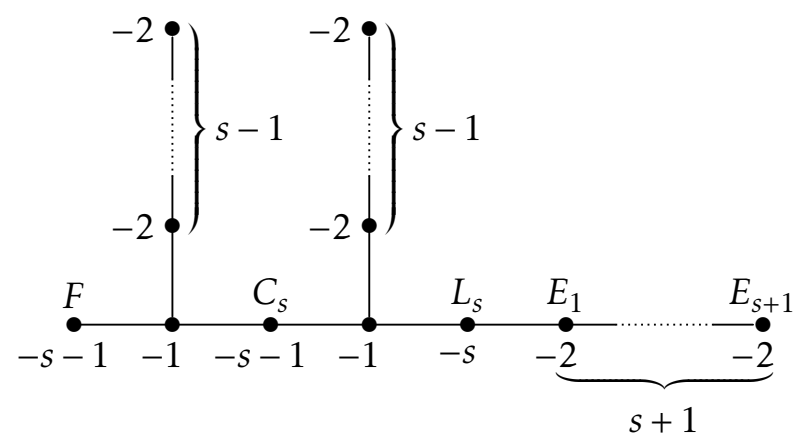

Figure 4: The weighted dual graph of the boundary divisor $B_{s}$.

Proposition 4.3. For every $s \geq 2, S_{s}$ is a fake real plane with contractible complexification and $\kappa\left(S_{s}\right)=$ $\kappa_{\mathbb{R}}\left(S_{s}\right)=2$. Furthermore, if $s, s^{\prime} \geq 2$ are two integers, then $S_{s}$ is birationally diffeomorphic to $S_{s^{\prime}}$ if and only if $s=s^{\prime}$.

Proof. The fact that the complexification of $S_{s}$ is contractible and of log-general type is proven in [18]: the log-general type is the purpose of the construction and the contrability is given in [18, Theorem 2]. Since $B_{s}=B_{s, \mathbb{R}}$ by construction, we have $\kappa_{\mathbb{R}}\left(S_{s}\right)=\kappa\left(S_{s}\right)=2$, and $S_{n}$ is a fake real plane by virtue of [5, Proposition 2.4]. By Proposition 4.2, every birational diffeomorphism between $S_{s}$ and $S_{s^{\prime}}$ is an isomorphism. But the description of the dual graphs of the boundaries $B_{s}$ in Figure 4 implies that every isomorphism between $S_{s}$ and $S_{s^{\prime}}$ extends to an isomorphism of pairs between $\left(V_{s}, B_{s}\right)$ and $\left(V_{s^{\prime}}, B_{S^{\prime}}\right)$ and that two such pairs are non isomorphic for different $s$ and $s^{\prime}$.

\section{C. Fake real planes of general type with nontrivial real forms}

To complete this section, we reconsider a family of fake real planes of general type with two real forms intoduced in [5, §3.2.2]. We start with the projective duals $\Gamma_{1}$ and $\Gamma_{2}$ of real nodal cubic curves $C_{1}, C_{2} \subseteq \mathbb{P}_{\mathbb{R}}^{2}$, such that the two branches at the singular point of $C_{1}, C_{2}$ are real, respectively non-real.

Note that $C_{1}, C_{2}$ are not equivalent under $\operatorname{Aut}\left(\mathbb{P}_{\mathbb{R}}^{2}\right)=\mathrm{PGL}_{2}(\mathbb{R})$, and that every real nodal cubic curve of $\mathbb{P}_{\mathbb{R}}^{2}$ is projectively equivalent to either $C_{1}$ or $C_{2}$. The latter can be checked by looking at the parametrisations $\mathbb{P}_{\mathbb{R}}^{1} \rightarrow C_{1}, C_{2}$, given by polynomials of degree 3 having the same value at two points, which are either real or pairs of non-real complex conjugates. Explicitely, one can choose, for instance, the equations of $C_{1}$ and $C_{2}$ to be

$$
x^{2} z-y^{2} z+x y^{2}=0 \text { and } x^{2} z+y^{2} z-x y^{2}=0 .^{2}
$$

With these coordinates, we find that $C_{1}$ and $C_{2}$ are exchanged by the non-real complex projective transformation $[x: y: z] \mapsto[x: \mathbf{i} y: z]$. Moreover, both curves $C_{1}, C_{2}$ have a singular point at $[0: 0: 1]$, an inflection point at $[0: 1: 0]$ and then two other complex inflection points, which are $\left[1: \pm \mathbf{i} \frac{\sqrt{3}}{3}: \frac{1}{4}\right]$ for $C_{1}$ and $\left[1: \pm \frac{\sqrt{3}}{3}: \frac{1}{4}\right]$ for $C_{2}$.

\footnotetext{
$2 \uparrow$ The equations given in $[5, \S 3.2 .2]$ are unfortunately false, as both are linearly equivalent, having one singular point with two real branches.
} 
The curves $\Gamma_{1}$ and $\Gamma_{2}$ are thus rational quartics with three cusps: an ordinary real cusp $p_{0}$ corresponding to the common $\mathbb{R}$-rational flex of $C_{1}$ and $C_{2}$, and either a pair of non-real conjugate cusps $q$ and $\bar{q}$ for $\Gamma_{1}$ or an additional pair or real ordinary cusps $q_{1}$ and $q_{2}$ for $\Gamma_{2}$. So $\Gamma_{1}$ and $\Gamma_{2}$ are not isomorphic over $\mathbb{R}$, but their respective complexifications are both projectively equivalent over $\mathbb{C}$. In fact, after change of coordinates, the curves $\Gamma_{1}$ and $\Gamma_{2}$ can be given by the equations

$$
\left(x^{2}+y^{2}\right)^{2}+z\left(2 x^{3}+2 x y^{2}-y^{2} z\right)=0 \text { and } x^{2} y^{2}+x^{2} z^{2}+y^{2} z^{2}+2 x y z(x+y-z)=0,
$$

and the projective transformation $\theta:[x: y: z] \mapsto[x+\mathbf{i} y: x-\mathbf{i} y: z / 2]$ maps $\Gamma_{1}$ isomorphically onto $\Gamma_{2}$. The cusps of $\Gamma_{1}$ are then $p_{0}=[0: 0: 1], q=[1: \mathbf{i}: 0], \bar{q}=[1:-\mathbf{i}: 0]$, and the ones of $\Gamma_{2}$ are $p_{0}=[0: 0: 1]$, $q_{1}=[1: 0: 0], q_{2}=[0: 1: 0]$.

For $i=1,2$, the tangent line $L_{i}=T_{p_{0}}\left(\Gamma_{i}\right)$ to $\Gamma_{i}$ at $p_{0}$ (given respectively by $y=0$ and $x=y$ and satisfying $\theta\left(L_{1}\right)=L_{2}$ ) intersects $\Gamma_{i}$ transversally in a unique other real point $p_{i}$ different from $p_{0}$ (being given by $p_{1}=[1: 0:-1 / 2]$ and $\left.p_{2}=[1: 1:-1 / 4]=\theta\left(p_{1}\right)\right)$. Let $(a, b)$ be a pair of positive integers such that $4 b-a= \pm 1$ and let $\tau_{i}: V_{i} \rightarrow \mathbb{P}_{\mathbb{R}}^{2}$ be the real birational morphism obtained by first blowing-up $p_{i}$ with exceptional divisor $E_{1} \simeq \mathbb{P}_{\mathbb{R}}^{1}$ and then blowing-up a sequence of real points on the successive total transforms of $E_{1}$ in such a way that the following two conditions are satisfied: a) the inverse image of $p_{i}$ is a chain of curves isomorphic to $\mathbb{P}_{\mathbb{R}}^{1}$ containing a unique (-1)-curve $A$ and b) the coefficients of $A$ in the total transform of $\Gamma_{i}$ and $L_{i}=T_{p_{0}}\left(\Gamma_{i}\right)$ are equal to $a$ and $b$ respectively. We denote the corresponding exceptional divisors by $E_{1}, \ldots, E_{r-1}, E_{r}=A$ and we let $B_{i}=\Gamma_{i} \cup T_{p_{0}}\left(\Gamma_{i}\right) \cup \bigcup_{j=1}^{r-1} E_{j}, i=1,2$.

Proposition 4.4. For every choice of integers $(a, b) \in \mathbb{Z}_{>0}$ as above, the following hold for the surfaces $S_{i}=V_{i} \backslash B_{i}$, $i=1,2$ :
a) $S_{1}$ and $S_{2}$ are $\mathbb{Z}$-acyclic fake real planes of Kodaira dimension 2 with isomorphic complexifications.
b) $\kappa_{\mathbb{R}}\left(S_{2}\right)=2$, in particular $S_{2}$ is not birationally diffeomorphic to $\mathbb{A}_{\mathbb{R}}^{2}$.
c) $\kappa_{\mathbb{R}}\left(S_{1}\right)=-\infty$, and $S_{1}$ is actually birationally diffeomorphic to $\mathbb{A}_{\mathbb{R}}^{2}$.

Proof. The complex surfaces $S_{1, \mathbb{C}}$ and $S_{2, \mathbb{C}}$ are isomorphic, by lifting the projective transformation $\theta$. The fact that $S_{1}$ and $S_{2}$ are $\mathbb{Z}$-acyclic fake real planes of log-general type is established in [5, Proposition 3.10]. Since by construction $B_{2}$ is a tree of $\mathbb{R}$-rational curves, we have $\kappa_{\mathbb{R}}\left(S_{2}\right)=\kappa\left(S_{2}\right)=2$ by Proposition 2.13 (2), and so $S_{2}$ is not birationally diffeomorphic to $\mathbb{A}_{\mathbb{R}}^{2}$. The fact that $S_{1}$ is birationally diffeomorphic to $\mathbb{A}_{\mathbb{R}}^{2}$ is proven in [6, Proposition 21].

\section{References}

[1] I. Biswas and J. Huisman, Rational real algebraic models of topological surfaces, Doc. Math. 12 (2007), 549-567. MR-2377243

[2] J. Blanc and F. Mangolte, Geometrically rational real conic bundles and very transitive actions, Compos. Math. 147 (2011), no. 1, 161-187. MR-2771129

[3] T. tom Dieck and T. Petrie, Contractible affine surfaces of Kodaira dimension one, Japan J. Math. 16 (1990), no. 1, 147-169. MR-1064448

[4] T. tom Dieck and T. Petrie, Homology planes and algebraic curves, Osaka J. Math. 30 (1993), no. 4, 855-886. MR-1250787

[5] A. Dubouloz and F. Mangolte, Fake real planes: exotic affine algebraic models of $\mathbb{R}^{2}$, Selecta Math. (N.S.) 23 (2017), no. 3, 1619-1668. MR-3663591 
[6] A. Dubouloz and F. Mangolte, Real Frontiers of Fake Planes, Eur. J. Math. 2 (2016), no. 1, 140-168. MR-3454095

[7] T. Fujita, On the topology of noncomplete algebraic surfaces, J. Fac. Sci. Univ. Tokyo Sect. IA Math. 29 (1982), no. 3, 503-566. MR-0687591

[8] R. V. Gurjar and M. Miyanishi, Affine surfaces with $\bar{\kappa} \leq 1$. In: Algebraic geometry and commutative algebra, Vol. I (in honor of M. Nagata), pp. 99-124, Kinokuniya, Tokyo, 1988. MR-0977756

[9] R. V. Gurjar and C. R. Pradeep, Q-homology planes are rational. III, Osaka J. Math. 36 (1999), no. 2, 259-335. MR-1736480

[10] R. V. Gurjar, C. R. Pradeep, and A. R. Shastri, On rationality of logarithmic $\mathbb{Q}$-homology planes. II, Osaka J. Math. 34 (1997), no. 3, 725-743. MR-1613057

[11] R. Hartshorne, Algebraic geometry, Graduate Texts in Mathematics, vol. 52, Springer-Verlag, New YorkHeidelberg, 1977. MR-0463157

[12] H. Hironaka, Resolution of singularities of an algebraic variety over a field of characteristic zero. I, II, Ann. of Math. (2) 79 (1964), 109-203; 205-326. MR-0199184

[13] J. Huisman and F. Mangolte, The group of automorphisms of a real rational surface is $n$-transitive, Bull. Lond. Math. Soc. 41 (2009), no. 3, 563-568. MR-2506841

[14] S. Iitaka, On D-dimensions of algebraic varieties, Proc. Japan Acad. 46 (1970), 487-489. MR-0285532

[15] S. Iitaka, On logarithmic Kodaira dimension of algebraic varieties. In: Complex analysis and algebraic geometry, pp. 175-189, Iwanami Shoten, Tokyo, 1977. MR-0569688

[16] S. Iitaka, Some applications of logarithmic Kodaira dimension. In: Proceedings of the International Symposium on Algebraic Geometry (Kyoto Univ., Kyoto, 1977), pp. 185-206, Kinokuniya Book Store, Tokyo, 1978. MR-0578859

[17] M. Miyanishi, Open Algebraic Surfaces, CRM Monograph Series, vol. 12, American Mathematical Society, Providence, RI, 2001. MR-1800276

[18] M. Miyanishi and T. Sugie, Examples of homology planes of general type. Appendix to: T. Sugie, On T. Petrie's problem concerning homology planes, J. Math. Kyoto Univ. 30 (1990), no. 2, 317-342. MR-1068794

[19] M. Nagata, Imbedding of an abstract variety in a complete variety, J. Math. Kyoto Univ. 2 (1962), 1-10. MR-0142549

[20] M. Nagata, A generalization of the imbedding problem of an abstract variety in a complete variety, J. Math. Kyoto Univ. 3 (1963), 89-102. MR-0158892

[21] C. P. Ramanujam, A topological characterisation of the affine plane as an algebraic variety, Ann. of Math. (2) 94 (1971), 69-88. MR-0286801

[22] R. Silhol, Real algebraic surfaces, Lecture Notes in Mathematics, vol. 1392, Springer-Verlag, Berlin, 1989. MR-1015720 